\title{
The effects of the global surface curvature on Makyoh-topography imaging
}

\author{
Ferenc Riesz* \\ Hungarian Academy of Sciences, Centre for Energy Research, Institute for Technical Physics and Materials \\ Science, P.O. Box 49, H-1525 Budapest, Hungary
}

Received November 27, 2018; accepted March 28, 2019; published March 31, 2019

\begin{abstract}
The effects of the global curvature of the reflecting surface on Makyoh (magic-mirror) topography imaging is analysed based on a geometrical optical model. It is shown that the effects can be taken into account by introducing an equivalent screen-to-sample distance which is a function of the real screen-to-sample distance and the global curvature. Special limiting cases are discussed and analysed for practical applications.
\end{abstract}

Makyoh (or magic-mirror) topography is a powerful topographic tool for qualitative visualisation of surface defects of semiconductor wafers [1]-[4] and other mirrorlike surfaces [5]. Makyoh topography gets its name from an ancient bronze mirror of the Far-East origin [6] (the word 'Makyoh' means 'magic mirror' in Japanese). Such a mirror has a backside relief pattern, which is transferred to the polished front face as a nearly invisible surface relief during the machining of the mirror. Projecting a parallel beam (e.g., sunlight) onto the front surface, a reflected pattern corresponding to the back pattern appears on a distant wall due to the focusing/defocusing action of the local curvatures of the surface relief (Fig. 1) [7], giving the illusion of transparency. The modern version works in a similar way: the studied surface is illuminated by a uniform-intensity collimated light beam, and a reflected image is formed on a screen placed some distance away from the sample. The resulting topogram thus reveals the surface defects and texture in the image in the form of dark/bright patches or regions. The practical implementations usually employ additional optical elements and electronic cameras for imaging instead of a simple screen [2].

The method's advantages, as compared to concurrent optical methods such as interferometry, are the extreme simplicity, low cost, no need for accurate sample positioning and calibration, real-time operation and high dynamic range. Makyoh topography found its most

\footnotetext{
*E-mail: riesz.ferenc@energia.mta.hu
}

fruitful applications in semiconductor technology for inline detection of surface defects [1]-[3], where qualitative detection is sufficient, and instant operation is required. Defected wafers thus could be removed from the manufacturing process to avoid faulted circuits and may be submitted to a more detailed and time-consuming individual analysis.

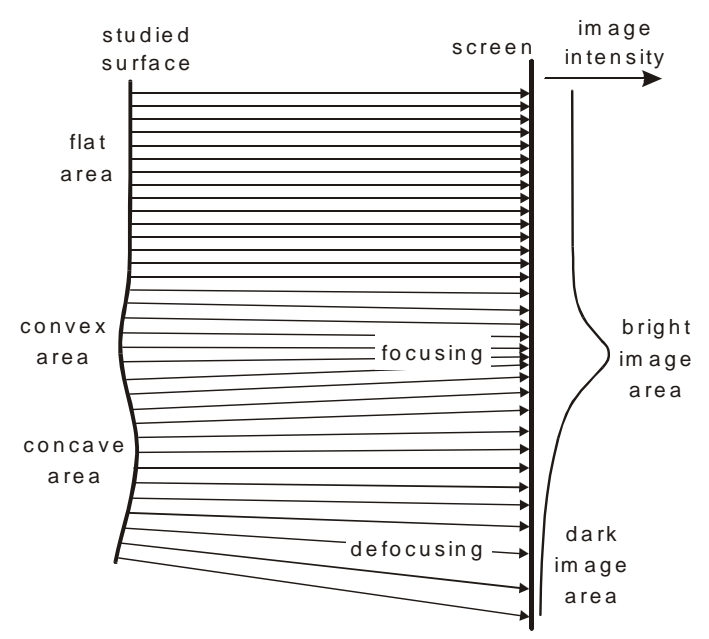

Fig. 1. Schematic reprsentation of Makyoh imaging

The studied samples often exhibit a global curvature. For example, in semiconductor technology the wafers are often curved due to deposited strained layers or possess a large-scale deformation. Ancient magic mirrors are also usually slightly convex [6]. The effects of this kind of global curvature on Makyoh imaging have, however, received only limited attention. Berry [8] has studied the special limiting case of a small surface relief. It was also shown that a low amount of curvature has a negligible effect on the method's sensitivity [9]. Therefore, the aim of the present paper is a comprehensive study of the effects of curvature. 
Although wave optical phenomena play in the role of the imaging mechanism [10], Makyoh imaging is understood usually in the framework of geometrical optics. A geometrical optical model of image formation has been presented in Ref. [11]. The two basic imaging equations are the following:

$$
\mathbf{f}(\mathbf{r})=\mathbf{r}-2 L \operatorname{grad} h(\mathbf{r})
$$

and

$$
I(\mathbf{f})=\frac{1}{\left|\left(1-2 L C_{\min }\right)\left(1-2 L C_{\max }\right)\right|}
$$

Equation (1) represents the mapping of a surface point $\mathbf{r}$ to the image point $\mathbf{f}$, according to the gradient field of the surface. The $I(\mathbf{f})$ intensities of the image points', as normalised to that of a flat surface, are determined by local curvatures, according to Eq. (2) - The reflectivity is assumed uniform. Here, $C_{\min }$ and $C_{\max }$ are the two principal curvatures in the point $\mathbf{r}$, that is, the minimum and maximum of the second derivatives of $h(\mathbf{r})$, the height profile. Positive curvatures mean a concave surface. $L$ is the basic imaging parameter, the screen-tosample distance. Increasing this distance increases the image contrast, thus, the sensitivity of the method, but also the amount of image distortion. An optimum $L$ value thus can be found, depending on the sample. We note that in the simple arrangement, $L$ is naturally positive, but it can be negative when additional optics are employed for imaging [12].

Consider now a surface with a uniform global isotropic curvature $C_{g}$. We wish to know this curvature's effects as compared to a globally flat surface. We assume that imaging is in the non-caustic regime (which is the preferred regime of Makyoh imaging [11]), that is, $1-2 L C_{\text {min,max }}>0$ for all over the surface; thus, we can drop the absolute signs from Eq. (2).

As for Eq. (1), it is evident that the added uniform curvature means a linearly varying slope added along the surface, so a linear magnification $1-2 L C_{g}$ of the original image occurs. This trivial effect will not be discussed further and no magnification is considered in the rest of the paper. We note that this phenomenon can be utilised to determine the sample curvature radius using structured illumination and a reference flat [13].

The effect of the global curvature on image intensities now can be treated as follows. We regard $C_{\min }$ and $C_{\max }$ as pertaining to an originally globally flat surface. Then, we substitute $C_{\min }+C_{g}$ and $C_{\max }+C_{g}$ into Eq. (2) for $C_{\min }$ and $C_{\text {max }}$. After performing the multiplications and combining the like terms, we obtain the following for the reciprocal of the resulting image intensity $I_{g}$ :

$$
\begin{gathered}
1 / I_{g}=1-2 L C_{\min }-4 L C_{g}-2 L C_{\max }+ \\
+4 L^{2} C_{\min } C_{\max }+4 L^{2} C_{\min } C_{g}++4 L^{2} C_{\max } C_{g}+4 L^{2} C_{g}^{2} .
\end{gathered}
$$

After rearranging, factoring out $\left(1-2 L C_{g}\right)^{2}$ and performing some grouping, we finally obtain:

$$
\begin{gathered}
\frac{1}{I_{g}}=\left(1-2 L C_{g}\right)^{2}\left[1-\frac{2 L}{1-2 C_{g}}\left(C_{\min }+C_{\max }\right)+\right. \\
\left.+\left(\frac{2 L}{1-2 L C_{g}}\right)^{2} C_{\min } C_{\max }\right]
\end{gathered}
$$

Comparing this formula to Eq. (2), we can conclude that the added $C_{g}$ overall curvature results in:

- an overall change in image intensity by a factor of $\left(1-2 L C_{g}\right)^{-2}$ and,

- the introduction of an equivalent surface-to-screen distance $L_{g}$ as

$$
L_{g}=\frac{L}{1-2 L C_{g}}
$$

instead of $L$.

A global intensity change is evident since magnification is also changed.

Slightly rearranging Eq. (5), $L_{g}$ can be expressed as a reciprocal addition of $L$ and $-1 /\left(2 C_{g}\right)$, the latter quantity being the focal length of the sample's mean surface interpreted as a spherical mirror. That is, a uniformly curved sample with a surface relief behaves as a flat sample with the surface relief "cascaded" with a curved mirror without a relief. Concave global curvature results in an increase of the equivalent $L$, while convex curvature decreases it.

The effects of the global curvature on imaging (contrast, patterns etc.) can thus be assessed by looking at the behaviour of the intensity with changing $L$. However, as the imaging is nonlinear [14] in both $L$ and the surface height profile, the actual behaviour depends on the relief pattern and the value of $L$.

It is instructive to examine three special limiting cases. Two of them represent the two cases when one of the two members of the reciprocal sum expressing $L_{g}$ is negligible compared to the other.

- $2\left|C_{g}\right|<|1 / L|$ : The global curvature is negligible, there is no noticable effect on imaging. This regime for the 
special case of a small surface relief was briefly discussed in Ref. [9].

- $2\left|C_{g}\right| \gg|1 / L|$ : in this case the term $1 / L$ is negligible ( $L$ is very large compared to the global curvature radius), thus $L_{g} \approx-1 /\left(2 C_{g}\right)$; that is, the resulting equivalent surface-to-screen distance will be independent of $L$. This results in the insensitivity of the projected screen pattern on the surface-to-screen distance (apart from the changing overall size); this feature is commonly observed [15] for ancient magic mirrors, since they are usually strongly convex. This limiting case was also discussed by Berry [8] but only for the special case of a small surface relief. This effect may also limit the sensitivity of Makyoh topography in a practical setting, as increasing $L$ does not lead to an increase of sensitivity.

- $2 C_{g}=1 / L$ : the screen is in the focus of the mean mirror surface. The image is in close proximity to the (diffraction-limited) focal point, with the surface deviations appearing as reflections scatttered around. This case is not suitable for topographic imaging, since the spatial position of the scattering areas is lost.

We stress finally that our analysis is valid for all surface shapes, since the original curvatures of the surface do not appear in the expression of $L_{g}$ and no approximations are introduced.

\section{References}

[1] K. Kugimiya, J. Cryst. Growth 103, 420 (1990).

[2] P. Blaustein, S. Hahn, Solid State Technol. 32, 27 (1989).

[3] Z.J. Pei, G.R. Fisher, M. Bhagavat, S. Kassir, Int. J. Machine Tools Manufacture 45, 1140 (2005).

[4] F. Riesz, Proc. SPIE 5458, 86 (2004).

[5] T. Hirogaki, E. Aoyama, R. Machinaka, H. Sueda, K. Ogawa, J. Japan Soc. Precision Eng. 73, 96 (2007).

[6] G. Saines, M.G. Tomilin, J. Opt. Technol. 66, 758 (1999).

[7] W.E. Ayrton, J. Perry, Proc. Roy. Soc. London 28, 127 (1878).

[8] M.V. Berry, Eur. J. Phys. 27, 109 (2006).

[9] F. Riesz, Optik 122, 2115 (2011).

[10] Z.J. Laczik, Opt. Eng. 39, 2562 (2000).

[11] F. Riesz, J. Phys. D: Appl. Phys. 33, 3033 (2000).

[12] F. Riesz, Rev. Sci. Instr. 72, 1591 (2001).

[13] J. Szabó, F. Riesz, B. Szentpáli, Japan. J. Appl. Phys. 35, L258 (1996).

[14] F. Riesz, J. Opt. 15, 075709 (2013).

[15] A.V. Gitin, Appl. Opt. 48, 1268 (2009). 